\title{
Modelo Baseado em Rede de Sensores Sem Fio Para Predição de Disfunções em Cultivos Agrícolas
}

\author{
Uélison Jean L Santos ${ }^{1}$, Rodrigo da Rosa Righi ${ }^{1}$, Cristiano André da Costa $^{1}$ \\ ${ }^{1}$ Programa de Pós-Graduação em Computação Aplicada - SoftwareLab - Unisinos \\ uelison.jean@gmail.com, rrrighi@unisinos.br, cac@unisinos.br
}

\begin{abstract}
One of the challenges for the future is to ensure food for all. However, the increase in food production is of concern, since most agricultural systems use environmental impacts resources. In this context, researches with IoT have shown great potential. However, there are still few viable options for application in the rural environment and that support the producer in the decision making. This article presents AgriPrediction, a model proposal that can be used by farmers using LoRa network architecture and predict dysfunctions using the ARIMA model. The results obtained during tests are positive since its application in arugula cultivation a $17.94 \%$ gain was obtained concerning leaf development and $14.29 \%$ in weight.
\end{abstract}

Resumo. Um dos desafios para o futuro é garantir alimento à todos. Porém, o aumento da produção de alimentos preocupa, uma vez que sistemas agrícolas utilizam recursos que causam impactos ambientais. Neste contexto, pesquisas com Internet das Coisas mostram alternativas, porém ainda existem poucas opções viáveis de aplicação no ambiente rural que auxiliem o produtor agrícola na tomada de decisões. Este artigo apresenta o AgriPrediction, uma proposta de modelo que pode ser aplicado por agricultores utilizando arquitetura de rede LoRa e predição de disfunções utilizando o modelo ARIMA. Os resultados obtidos são positivos, pois em sua aplicação em cultivo de rúcula obteve-se ganho de $17.94 \%$ em relação ao desenvolvimento das folhas e $14.29 \%$ no peso.

\section{Introdução}

Conforme [DESA 2017] a população mundial irá atingir em 2050 quase 10 bilhões de pessoas, tendo como desafio a produção de alimentos para todos. Este autor aponta como alternativas o uso de práticas sustentáveis e o aumento da produtividade, reduzindo assim os custos no uso de energia e água. Para atingir este objetivo ressalta-se a relevância de pesquisas voltadas para o auxílio da produção agrícola com a utilização da predição.

[Mohapatra and Lenka 2016] ressaltam que existe grande potencial em aplicações de Redes de Sensores sem Fio na área de Agricultura. Os autores relatam, no que tange a previsão em cultivos, que apenas um trabalho foi encontrado, refletindo a necessidade de futuros trabalhos nesta temática. Sugerem ainda pesquisas na área de monitoramento da vitalidade da plantação e previsão de rendimento utilizando serviços móveis e redes de sensores.

Uma vez analisados trabalhos relacionados à temática desta pesquisa, identificouse que ainda faltam estudos que possam ser aplicados de fato em um cultivo prático. Por meio da revisão da literatura percebeu-se que são utilizadas tecnologias de comunicação 
que suportam no máximo poucas centenas de metros, sendo que um cultivo geralmente é realizado em áreas que ocupam hectares e, por vezes, sem nenhum tipo de conectividade [Tripathy et al. 2011, Sawant et al. 2014, Liu et al. 2016, Mohapatra and Lenka 2016].

Para contribuir com o aumento da produtividade da produção agrícola, por meio da aplicação de novas tecnologias à agricultura de precisão, propõe-se o modelo AgriPrediction. O modelo utiliza sensores distribuídos pelo campo para obter informações de um cultivo. Estas informações trafegam por uma rede de sensores sem fio (WSN) até uma aplicação onde são analisados utilizando o modelo de previsão ARIMA. Os dados são analisados e comparados com valores pré-estabelecidos pelo agricultor, caso os valores previstos pelo ARIMA estejam fora dos ideais, notifica-se o agricultor através de um dispositivo móvel, possibilitando a tomada de ações de forma a prevenir perdas. Objetivando analisar o modelo proposto, construiu-se uma estufa para o cultivo de rúcula (Eruca sativa), hortaliça escolhida por seu rápido desenvolvimento.

No decorrer desta pesquisa estão apresentados os trabalhos relacionados (Seção 2); o modelo AgriPrediction (Seção 3); a metodologia de avaliação (Seção 4); a análise da aplicação do modelo (Seção 5) e por fim, sua conclusão, bem como contribuição científica do trabalho e desafios futuros.

\section{Trabalhos Relacionados}

[Tripathy et al. 2011] realizaram um experimento para entender as relações entre doenças/pestes e clima utilizando sensoriamento sem fio com foco no Inseto Tripé e na peste Necrose do Broto. Já [Sawant et al. 2014] propuseram uma rede sem fio para integração de sistemas de sensoriamento voltado para agricultura de precisão, a qual denominou KrishiSense. [Liu et al. 2016], trabalharam em um novo método de previsão baseado em um algoritmo Extreme Learning Machine (ELM) utilizado para prever a temperatura e a umidade em um ambiente de estufa prático em Nanjing, China.

[Yuhan et al. 2016] desenvolveram um experimento com plantas de tomateiro em uma estufa para modelagem de predição de taxa fotossintética. Os autores empregaram redes neurais para melhorar a precisão da solução. [Mohapatra and Lenka 2016] concentraram-se em duas estratégias de otimização utilizadas para predizer a necessidade horária de CM no solo. [Edwards-Murphy et al. 2016] propuseram o modelo b + WSN, uma Rede de Sensores sem Fio heterogênea para coletar dados usando uma gama de parâmetros de uma colméia.

A Tabela 1 compara os artigos citados quanto a proposta de trabalho, coleta, exibição e análise dos dados, conectividade e localização dos sensores e local do experimento. Uma vez analisados os trabalhos relacionados, identificou-se que apesar de alguns pesquisadores estarem atuando com WSN na agricultura, ainda faltam estudos aplicados a um cultivo prático. As pesquisas utilizam tecnologias de comunicação que suportam poucas centenas de metros quando um cultivo geralmente é realizado em áreas que ocupam hectares, como já mencionado anteriormente.

Ressalta-se que poucos autores realizaram análises aprofundadas dos dados, desta forma, esta pesquisa se preocupa em fornecer não apenas dados do cultivo, mas possibilitar a tomada de decisões assertivas frente aos possíveis problemas. Além disto, os autores se limitam a realizar ações reativas somente quando algum limite é rompido. A tomada 
Tabela 1. Compartivo dos trabalhos Relacionados

\begin{tabular}{|c|c|c|c|c|c|c|}
\hline Artigo & Análise & Rede & Dados & Visualização & Localização & Aplicação \\
\hline [Tripathy et al. 2011] & Mineração & $\begin{array}{l}\text { ZigBee, } \\
\text { GPRS }\end{array}$ & Manual & N/A & India & Amendoin \\
\hline [Sawant et al. 2014] & N/A & Wi-Fi & Agendado & Twitter & India & Laranja \\
\hline [Liu et al. 2016] & ELM & ZigBee & N/A & N/A & China & Simulação \\
\hline [Yuhan et al. 2016] & $\begin{array}{l}\text { Rede } \\
\text { Neural } \\
\text { BP }\end{array}$ & N/A & Agendado & N/A & China & Tomate \\
\hline [Mohapatra and Lenka 2016] & $\begin{array}{l}\text { Rede } \\
\text { Neural }\end{array}$ & $\begin{array}{l}\text { Zigbee, } \\
\text { Wi-Fi }\end{array}$ & Agendado & N/A & India & Grama \\
\hline [Edwards-Murphy et al. 2016] & $\begin{array}{l}\text { Árvore } \\
\text { de } \\
\text { decisão }\end{array}$ & $\begin{array}{l}\text { ZigBee, } \\
3 \mathrm{G}\end{array}$ & Agendado & $\mathrm{N} / \mathrm{A}$ & Ireland & Apicultura \\
\hline
\end{tabular}

de ação de forma preditiva é melhor se comparada a reativa, pois, não exige que os limites sejam rompidos, mantendo o cultivo sempre dentro dos parâmetros.

\section{Modelo AgriPrediction e Decisões do Projeto}

De forma a possibilitar a aplicação do modelo em cenário real para que auxilie o produtor na previsão de problemas foram tomadas as seguintes decisões:

Estrutura de comunicação WSN: Optou-se pela utilização de Wireless Sensor Network (WSN) para coleta de dados do plantio e para garantir a conectividade entre os nós de sensores para transmitir os dados coletados dos nós para o servidor processá-los.

Tecnologia de WSN LoRa: O modelo propõe a construção de um link de rede entre as estações de coleta de dados e um Gateway utilizando a rede LPWAN LoRa ${ }^{1}$. Uma rede LoRa pode ter alcance de até $40 \mathrm{~km}$ em campo aberto o que possibilita a real aplicação da solução proposta [Alliance 2015]. Além disto, LoRa é um protocolo de baixo consumo de energia, não necessita nenhum tipo de assinatura para uso e opera em frequências liberadas. Possui tráfego de dados adaptativos e é possível a conexão de milhões de nós, ficando a limitação no hub da rede. Características que possibilitam a sua utilização com os requisitos necessários para operar no campo.

Predição ARIMA: São instrumentos de previsão cujo foco não está na construção de um modelo com equações únicas ou simultâneas, mas sim, na análise das propriedades probabilísticas das séries temporais, e assim, não são embasados em teorias econômicas [GUJARATI 2006].A decisão de utilizar este modelo deu-se pelo fato de que os dados obtidos por esta pesquisa foram coletados sequencialmente ao longo do tempo, necessitando de uma análise de série temporal tendo o modelo apresentado resultados positivos em pesquisas com sua utilização.

\subsection{Arquitetura}

A arquitetura do AgriPrediction foi dividida em AgriMesure, responsável pela coleta das informações; Servidor AgriPrediction, responsável por armazenar as informações, hospedar os serviços de predição, notificação e a interface; Gateway, responsável por receber

\footnotetext{
${ }^{1}$ https://www.lora-alliance.org
} 
e encaminhar as informações dos AgriMesures para o Servidor AgriPrediction e dispositivo móvel, que recebe as notificações. A Figura 1 exibe a representação do Modelo AgriPrediction.

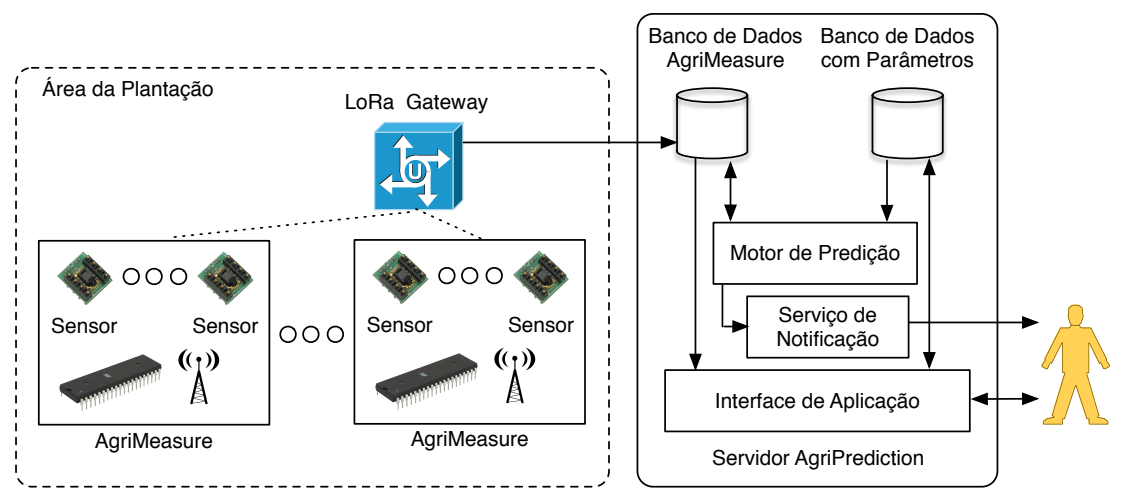

Figura 1. Arquitetura do AgriPrediction.

Os dados do cultivo são lidos por meio de uma estação de leitura de sensores denominada AgriMesure. Cada estação é composta de sensores; módulo de conectividade sem fio LoRa; micro controlador; placa solar; bateria e um identificador único de zona. $\mathrm{O}$ identificador de zona pode ser o ID do dispositivo, visando um menor custo, ou coordenadas obtidas através de uma placa de posicionamento GPS. O micro controlador é utilizado como solução para transformar os dados digitais e analógicos colhidos dos sensores para informações utilizáveis. Além disto, ele é responsável por enviar os dados através do chip de transmissão LoRa. A arquitetura do AgriMesure é voltada para micro controladores open hardware.

Os sensores utilizados podem medir umidade, temperatura, luminosidade, radiação solar, condutividade elétrica do solo, $\mathrm{PH}$, clorofila da planta entre outros parâmetros, basta configurar o sensor utilizado em uma entrada do micro controlador, que o mesmo encaminha as informações até um gateway LoRa. O Gateway recebe os dados no protocolo LoRa e os encaminha ao sistema de armazenamento de dados. Uma vez armazenados os dados, o sistema responsável por executar o cálculo do modelo ARIMA irá incluir as informações recentes. O resultado do cálculo é então comparado com os dados de referência inseridos pelo agricultor na interface da aplicação. Caso os valores previstos superem os limites inferiores ou superiores do cultivo em questão, o sistema de notificação é acionado informando o agricultor para que o mesmo possa tomar a ação. Pela interface da aplicação o agricultor pode acompanhar como estão os valores dos sensores utilizados, bem como configurar os valores de referência e o tempo de predição futuro em horas.

Para tornar o projeto viável financeiramente toda a arquitetura do projeto pode ser construída utilizando dispositivos open hardware. Por fim, considerando que o ambiente é de suma importância, é implementada uma topologia de rede estrela utilizando protocolo LoRa, pois este protocolo além de baixo consumo de energia possibilita a comunicação em até $40 \mathrm{~km}$. Isto torna a arquitetura viável para utilização no campo, onde fazendas possuem vários hectares. 


\subsection{Estratégia de Predição}

O mecanismo de previsão apresentado no servidor funciona de acordo com o modelo de predisão ARIMA. É adequado quando temos uma das seguintes situações: (i) quando a série temporal apresenta uma tendência; (ii) quando a série temporal não apresenta um componente sazonal, mas apenas o componente tendência; (iii) quando a série temporal é classificada como não estacionária e homogênea. O ARIMA considera a tendência de séries temporais com um triplo $(p, d, q)$, onde $p$ é o número de termos autorregressivos, $d$ é o número de diferenças e $q$ é o número de termos e pode ser representado por $\Theta(B)(1-$ $B)^{d} Y_{t}=\Theta(B) a_{t}$ [Sáfadi 2004].

Ao iniciar sua execução, o serviço de predição carrega as informações obtidas pelos AgriMesures que estão armazenadas dentro do banco de dados, sendo o mesmo responsável por organizar as séries dos dados coletados por cada sensor conectado. Por definição, somente as informações das últimas 24 horas são utilizadas, de forma a evitar tendências de valores do passado no cálculo da predição. Uma vez organizados os dados de cada sensor de cada AgriMesure em suas séries iniciam-se os cálculos da predição. Ao executar o modelo, tem-se como resultado um valor máximo e um valor mínimo previsto para um dado momento no futuro. Por padrão, o modelo AgriPrediction define a previsão para a próxima hora, mas este parâmetro pode ser alterado pelo usuário.

\section{Metodologia de Avaliação}

Cada AgriMesure (estação de sensores) possui medição de temperatura e umidade do ar e do solo, luminosidade e um segundo sensor de temperatura externo, sendo este disposto fora da estufa de cultivo. Para medição da temperatura externa, o sensor ds18b20 foi utilizado. Este sensor trabalha com tensão de operação de 3 a 5,5V, possuindo atuação de $-55^{\circ} \mathrm{C}$ a $+125^{\circ} \mathrm{C}$. Sua precisão é de $0,5^{\circ} \mathrm{C}$ entre $-10{ }^{\circ} \mathrm{C}$ a $+85^{\circ} \mathrm{C}$. Para possibilitar seu uso externo o mesmo possui ponta de aço inoxidável de 6 x $50 \mathrm{~mm}$ e é a prova d'água. Para transmissão de dados foi utilizado o protocolo One Wire.

Para medir a umidade e temperatura do solo, o sensor SHT10 foi utilizado em um encapsulamento de malha de metal para permitir seu uso sem danificá-lo. Este sensor opera com uma tensão de operação de $2,2 \mathrm{~V}$ a $5,5 \mathrm{~V}$ e permite a medição de umidade de 0 a $100 \% \mathrm{RH}$, com uma precisão de $4,5 \%$ e medição de temperatura de $-40^{\circ} \mathrm{C}$ a $120^{\circ}$ C com $0,5 \%$ de precisão. Sua transmissão de dados ocorre de forma digital, através de comunicação serial. Para medir a luminosidade, o sensor TSL 2561, medindo de 0,1 a 45.000 lux, foi aplicado. Este sensor se comunica via protocolo I2C, consumindo 0,6 mA usando uma fonte de $3 \mathrm{~V}$.

Um Arduino Uno é utilizado para comunicação com os sensores, possui 14 pinos de entrada ou saída, dos quais 6 podem ser utilizados como saídas PWM e 6 entradas analógicas, controlados por um microcontrolador ATmega328 com clock de $16 \mathrm{MHz}$ suportando tensão de entrada de 6 a $20 \mathrm{~V}$ e opera com 5V. Para alimentar o sistema, é utilizada uma placa solar de $6 \mathrm{~V}$ de $3.8 \mathrm{~W}$ e uma bateria auxiliar de $6 \mathrm{~V}$ de capacidade de 4.5Ah. Cada AgriMesure possui uma placa de comunicação LoRa que envia informações para o gateway LoRa. A placa utiliza o módulo SX1272 da Microchip e está conectada a uma antena omnidirecional de $3 \mathrm{dbi}$. A frequência utilizada para comunicação é $868 \mathrm{MHz}$. Este módulo possui sensitividade de $-134 \mathrm{dBm}$ e possui alcance de $21 \mathrm{~km}$ em campo aberto 
com enlace de linha visada e $2 \mathrm{~km}$ em ambientes sem enlace de linha visada. A Figura 2 apresenta o protótipo do AgriPrediction.

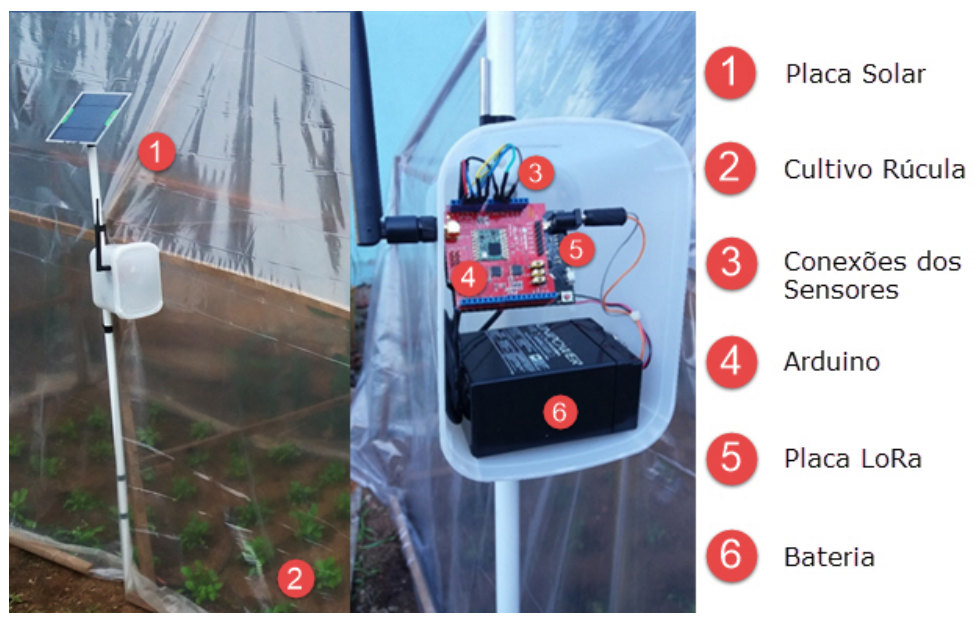

Figura 2. Protótipo do AgriPrediction

Para concepção do Gateway foi utilizado um Arduino Mega conectado a um módulo LoRa SX1272 de 868MHz. Este foi escolhido por possuir maior capacidade de memória e processamento para receber e processar os dados. Em seu software, foi configurado o módulo SX1272 com auxílio da biblioteca SX1272.h, distribuída pela Libelium de forma gratuita. Com o uso da biblioteca foi possível configurar o módulo LoRa e o Arduino para operar como receptor de mensagens, recebidas no primeiro canal da frequência $868 \mathrm{MHz}$. Para comunicação com o SX1272 a biblioteca utiliza os pinos de SPI do Arduino para comunicação serial. Os dados recebidos pelo gateway através da placa LoRa são encaminhados para o servidor por comunicação serial, e no servidor são armazenados em arquivo. Para comunicação, uma antena externa omnidirecional de $2 \mathrm{dbi}$ foi conectada ao módulo LoRa, montada em uma haste de 3 metros.

\subsection{Implementação de Predição e Notificações}

$\mathrm{O}$ arquivo contendo as leituras dos AgriMesures é lido pelo Software $\mathrm{R}$ a cada cinco minutos, onde o mesmo obtém as linhas referentes as últimas $24 \mathrm{H}$ de todos os nós. O R é um software estatístico desenvolvido por Ross Ihaka e Robert Gentleman. É ao mesmo tempo um ambiente de trabalho e uma linguagem de programação, que é escrita utilizando código descritivo. Os resultados são exibidos em formato texto ou exibição gráfica. É um software gratuito e de código aberto e suporta sistemas operacionais Linux, Mac OS e Windows [de Micheaux et al. 2013].

O script desenvolvido para o R é responsável pela separação das informações e executa o modelo ARIMA para cada um dos valores obtidos pelos sensores do AgriMesure, obtendo a previsão de 10 próximos valores. Uma média destes valores é calculada e comparada com os valores preestabelecidos preenchidos previamente, presentes em um arquivo separado. Baseado nesta comparação o script verifica se é necessário informar um possível desvio. Caso necessário, as notificações são realizadas com a utilização da biblioteca pushoverr, que possibilita enviar uma notificação para um dispositivo móvel configurado com o aplicativo Pushover. Os valores obtidos pela predição são armazena- 
dos em um outro arquivo, possibilitando o rastreio das informações. A Figura 3 exibe as notificações recebidas por smartphone dos eventos ocorridos durante o experimento.

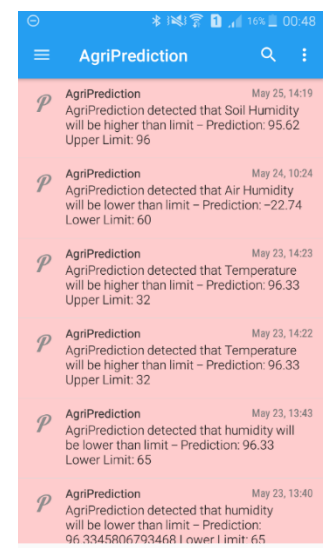

Figura 3. Exempo de Notificações recebidas em Smartphone

\subsection{Cultivo de Rúcula}

Selecionamos a rúcula como nossa cultura agrícola alvo, por ser um vegetal de fácil acesso e crescimento relativamente rápido, aproximadamente 30 a 40 dias após a semeadura [LINHARES 2007]. A área plantada mediu 3 metros de largura por 4 metros de comprimento e foi composta por uma estrutura de madeira e cobertura de filme plástico transparente, transformando-a em estufa. Foram plantadas um total de 100 mudas, semeadas na mesma data e uma semana após a germinação. Antes do plantio, o solo foi lixado por 20 centímetros e teve aplicação de lona plástica para permitir o controle da umidade do solo.

A estufa foi fisicamente dividida para a análise do modelo. De um lado, as ações foram tomadas ao longo da cultura para manter o plantio dentro dos parâmetros, enquanto o outro lado só foi regado a cada dois dias. O sensore de umidade e temperatura do solo foi posicionado a $10 \mathrm{~cm}$ da haste AgriMesure, enquanto o sensor de temperatura e umidade do ar foi disposto ao longo da mesma haste. O sensor de temperatura externo esteve abaixo da placa solar que estava no topo da haste em um ângulo de 45 graus. A Figura 4 ilustra $o$ cultivo utilizado durante o experimento. Finalmente, o Arduino e a placa LoRa estavam localizados dentro de uma caixa de plástico protegida do clima. As conexões entre os dispositivos foram feitas por meio de fio elétrico compatível. O experimento foi realizado na cidade de Esteio, RS, em um local com clima ameno e sombra durante a maior parte do dia.

\section{Análise da aplicação do AgriPrediction}

Para testar o modelo, a estufa foi dividida em duas seções, sendo O cultivo mantido por três semanas. Então, realizou-se a coleta aleatória de cinco plantas de cada lado para diagnóstico. Cada planta foi pesada com uma balança de precisão e teve suas cinco maiores folhas medidas com um paquímetro, considerando-se a média das folhas. No decorrer do cultivo um dos lados recebeu água a cada dois dias (Lado A), enquanto o outro recebeu água conforme a necessidade monitorada, tendo umidade e temperatura controladas através da abertura ou fechamento da estufa e controle de incidência de luz (Lado B). O 


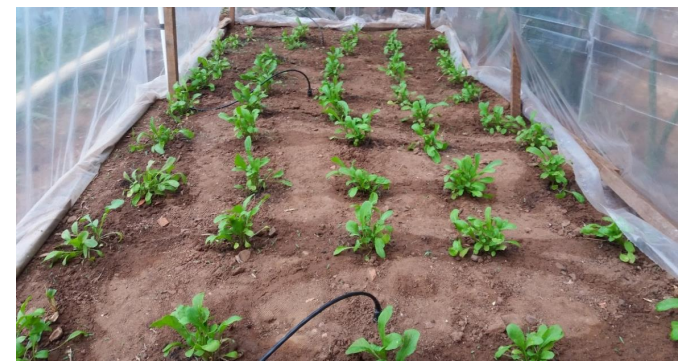

Figura 4. Estufa e sensores utilizados no experimento

Gateway foi posicionado a 15 metros da plantação em um ambiente fechado com barreiras de construções entre a plantação e com sinal SNR em média de $8 \mathrm{dBi}$. Este foi conectado a um servidor que estava executando o script desenvolvido para $\mathrm{R}$, responsável por obter os dados coletados pelo Gateway e aplicar a predição nos dados.

Um smartphone Android foi configurado para receber as notificações através do aplicativo Pushover. Os valores configurados como referência do cultivo foram de $16^{\circ} \mathrm{C}$ a $25^{\circ} \mathrm{C}$ de temperatura local e de $50 \%$ a $80 \%$ de umidade no ar; para o sensor no solo os valores foram de $14^{\circ} \mathrm{C}$ a $24^{\circ} \mathrm{C}$ e de $80 \%$ a $90 \%$ de umidade; por fim, o sensor de temperatura externo foi configurado de $15^{\circ} \mathrm{C}$ a $24^{\circ} \mathrm{C}$. Os sensores AgriMesures foram ligados e o Gateway conectado utilizando um cabo USB e interface serial a um computador executando o software CoolTerm que recebe dados por uma porta serial e armazena em arquivo texto. Um script desenvolvido para o software $\mathrm{R}$ foi executado para ler as informações do arquivo de texto. Durante sua execução ele ignora as linhas iniciais do arquivo, que são de controle do Gateway, lê o conteúdo, organiza as informações dos sensores em objetos de séries temporais, executa o cálculo do modelo. Abaixo apresenta-se os resultados com e sem o uso do AgriPrediction.

Sem o AgriPrediction: As folhas obtiveram um tamanho médio de 7,67 cm e um peso médio de $0,0035 \mathrm{Kg}$. Neste experimento, nenhuma ação foi tomada contra os valores verificados pelos sensores AgriPrediction. Na Figura 5, podemos ver um gráfico do décimo dia de monitoramento da temperatura do solo deste experimento, onde a temperatura acabou por ultrapassar os limites pré-definidos (valores de referência ou limiares).

Com o AgriPrediction: As plantas obtiveram um tamanho médio de $9,04 \mathrm{~cm}$ e peso médio de $0,004 \mathrm{Kg}$. Observa-se aqui que ao utilizar o monitoramento e realizar as ações sugeridas propostas pelo AgriPrediction foi possível obter um ganho de 17,94\% quanto ao tamanho das folhas e 14,29\% quando considerado o peso das plantas. A ideia é manter os legumes no chão sempre em condições ideais. Sempre que o algoritmo de previsão indicar um valor futuro fora das condições de cultivo seguras uma notificação ocorrerá no smartphone do agricultor, de modo que ações necessárias possam ser tomadas para corrigir/mitigar os problemas. Regar o plantio e controlar a umidade do ar representam as principais ações que fizemos neste experimento. A figura 6 mostra dados sobre a umidade do solo no décimo dia do experimento ao permitir as ações do AgriPrediction.

\section{Conclusão}

Este artigo apresentou um modelo de arquitetura de Redes de Sensores sem Fio (AgriPrediction) utilizando protocolo de rede LoRa para ser empregado por agricultores na 


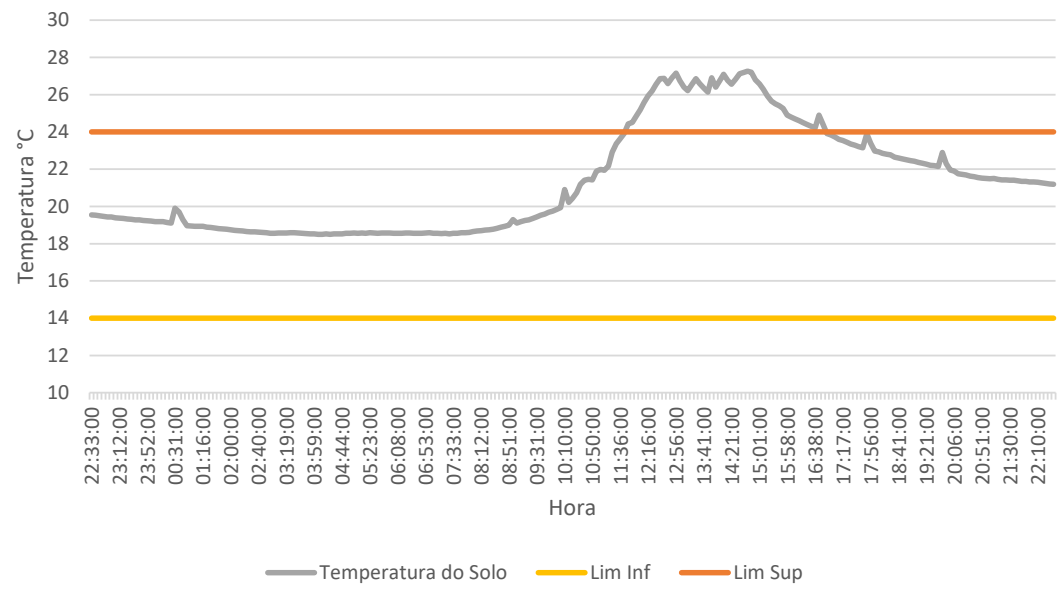

Figura 5. Temperatura do solo em experimento sem usar previsão e notificações.

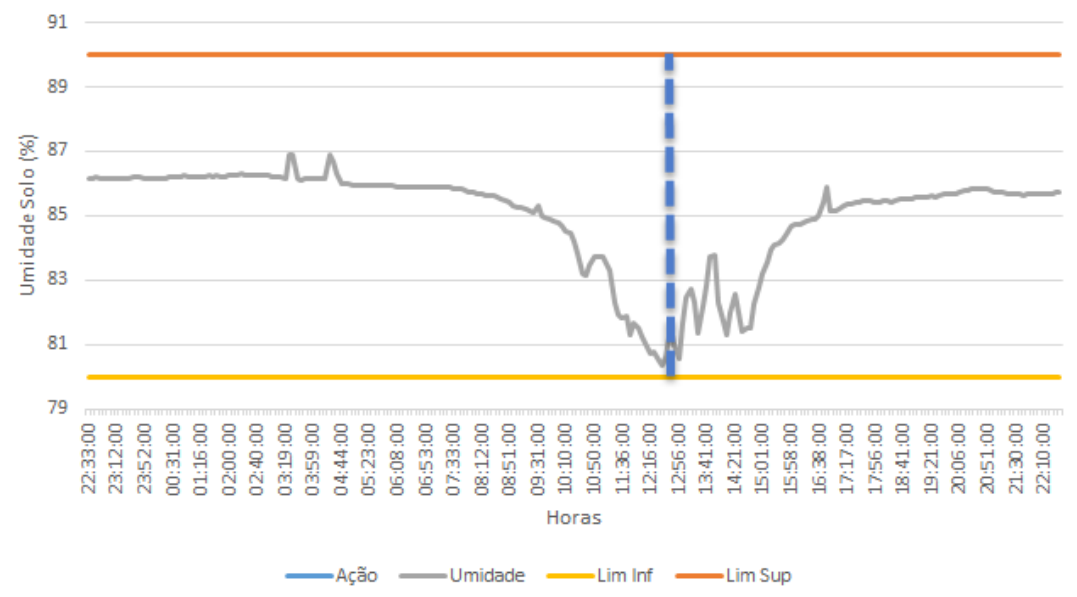

Figura 6. Umidade do solo e a interação do agricultor para corrigir eventuais problemas depois de receber uma notificação AgriPrediction.

implementação de Agricultura de Precisão e Predição. Diferente dos trabalhos relacionados, o AgriPrediction trabalha com uma tecnologia que possibilita o seu uso em situações reais em conjunto com algoritmo de predição. Deste modo, possibilita a previsão de problemas, bem como a tomada de ações ativas. Sua contribuição está na área da agricultura combinando redes de sensores de curta e média cobertura e o modelo de predição ARIMA para o lançamento de ações corretivas e melhor rendimento de cultivos agrícolas.

Verificou-se que com a utilização do Arduino, chip LoRa SX1272, sensores SHT10, ds18b20 e TSL 2561 aplicados ao cultivo, obteve-se sucesso nos testes de envio e recebimento de mensagens LoRa, aplicação do modelo ARIMA e ganho de $17.95 \%$ no desenvolvimento das folhas e $14.29 \%$ no peso das plantas.

Os resultados obtidos sinalizam a viabilidade da proposta, entretanto, os autores reconhecem as limitações. Quanto aos trabalhos futuros pode-se incluir a criação de aplicativo para celular que possibilite o acompanhamento em tempo real do cultivo, além do desenvolvimento de mecanismo de segurança para a transmissão dos dados e testes em cultivos. Além disso, o modelo proposto tem como alvo a área da agricultura, porém, objetiva-se estender os testes do mesmo no contexto de cidades inteligentes, para monito- 
ramento do trânsito.

\section{Agradecimentos}

Este trabalho é parcialmente financiado pelos seguintes órgãos de pesquisa brasileiros: CNPq, Fapergs e CAPES.

\section{Referências}

Alliance, L. (2015). Lorawan ${ }^{\mathrm{TM}}$ specification. LoRa Alliance.

de Micheaux, P. L., Drouilhet, R., and Liquet, B. (2013). The R Software. Springer.

DESA, U. (2017). World population prospects: The 2017 revision, key findings and advance tables. Working PaperNo.

Edwards-Murphy, F., Magno, M., Whelan, P. M., O'Halloran, J., and Popovici, E. M. (2016). b+ wsn: Smart beehive with preliminary decision tree analysis for agriculture and honey bee health monitoring. Computers and Electronics in Agriculture, 124:211219.

GUJARATI, D. (2006). N. econometria básica. $3^{\text {a }}$ edição.

LINHARES, P. C. F. (2007). Produção de rúcula em função de diferentes quantidades e tempos de decomposição de jitirana. $\mathrm{PhD}$ thesis, Dissertação (Mestrado em Fitotecnia)-Universidade Federal Rural do Semi-Árido. Mossoró, RN.

Liu, Q., Jin, D., Shen, J., Fu, Z., and Linge, N. (2016). A wsn-based prediction model of microclimate in a greenhouse using extreme learning approaches. In Advanced Communication Technology (ICACT), 2016 18th International Conference on, pages 730-735. IEEE.

Mohapatra, A. G. and Lenka, S. K. (2016). Neural network pattern classification and weather dependent fuzzy logic model for irrigation control in wsn based precision agriculture. Procedia Computer Science, 78:499-506.

Sáfadi, T. (2004). Use of time series analysis for the water outflow at furnas dam. Ciência e Agrotecnologia, 28(1):142-148.

Sawant, S. A., Adinarayana, J., and Durbha, S. S. (2014). Krishisense: A semantically aware web enabled wireless sensor network system for precision agriculture applications. In Geoscience and Remote Sensing Symposium (IGARSS), 2014 IEEE International, pages 4090-4093. IEEE.

Tripathy, A., Adinarayana, J., Sudharsan, D., Merchant, S., Desai, U., Vijayalakshmi, K., Reddy, D. R., Sreenivas, G., Ninomiya, S., Hirafuji, M., et al. (2011). Data mining and wireless sensor network for agriculture pest/disease predictions. In Information and Communication Technologies (WICT), 2011 World Congress on, pages 1229-1234. IEEE.

Yuhan, J., Yiqiong, J., Ting, L., Man, Z., Sha, S., and Minzan, L. (2016). An improved method for prediction of tomato photosynthetic rate based on wsn in greenhouse. International Journal of Agricultural and Biological Engineering, 9(1):146. 